\title{
Sexual Dysfunction
}

Jean D. Koebler

I. Background Information

II. Sexual Functioning

A. The Sexual Response Cycle

III. Establishing the Diagnosis: Sexual Dysfunctions and Problems

A. Definitions and Physical Etiologies

B. Other Sexual Problems

C. Male Sexual Dysfunction

D. Psychological Causes of Sexual Dysfunction
IV. Management of Sexual Problems

A. Sexual History

B. Office Management of Sexual Dysfunction

C. Referral

V. Case Study

VI. Summary

A. Reading for the Female Patient

B. Additional Suggested Web Sites

VII. Suggested Reading

\section{Background Information}

Female sexual dysfunction or dissatisfaction is a common complaint among obstetric-gynecology patients. These complaints may result from medical treatments and procedures, or may secondarily complicate the management and patient compliance of treatment for other medical issues. Therefore, it is important that clinicians treating females obtain basic information regarding human sexual function and dysfunction. This chapter presents an integration of the clinical research as well as the author's experience regarding sexual function and the management of sexual problems often presented in an obstetricgynecology practice.

\section{Sexual Functioning}

\section{The Sexual Response Cycle}

Sexual functioning is often described through the sexual response cycle, which includes sexual drive (libido), arousal, orgasm, and resolution. Each phase interacts with and influences the presence or absence of the other, and does not necessarily proceed in a linear fashion. Each is affected by psychological, 
sociological, and relational processes. However, natural sexual function throughout the whole sexual response cycle is physiologically dependent on adequate hormone levels - particularly estrogen and testosterone, enzymes that convert precursor hormones to the above-mentioned hormones, nonhormonal neurotransmitters like dopamine, adequate blood supply, and adequate central and peripheral nerve conduction. The clinician who assesses all of the physiologic and nonphysiologic areas, when presented with a patient with sexual dysfunction, will obtain a better diagnosis and treatment plan.

Sexual drive is the urge to obtain sexual stimulation. It can take the form of an urge to masturbate, watch or hear erotica, experience sexual dreams, or engage in stimulation with another person.

Sexual arousal occurs in two basic forms: mental and physiologic. Mental arousal occurs when a person reports feeling "turned on" or pleasure to erotic stimuli. Physiologic arousal usually includes genital vasocongestion, vaginal lubrication, increased genital erotic sensitivity, and the presence of prostatic-type secretions in the bladder and urethra as well as increases in respiration, pulse, and blood pressure. Mental arousal and physiologic arousal do not always occur together, as is sometimes the case with rape or unwanted stimulation, where vaginal lubrication may occur without any sense of subjective arousal. Physical reactions of sexual arousal in the female are presented in Table 20.1. Not all of these are observed in every woman.

The orgasmic phase of the sexual response cycle mainly consists of the 0.8 -second involuntary contractions of the vaginal canal and uterus, accompanied by a subjective sense of sudden heightened pleasure. Some

\section{Table 20.1. Physical reactions during female sexual arousal.}

1. Swelling of the labia, clitoris, and introitus

2. Lubrication of the vaginal canal-a "sweating phenomenon" through the mucous membranes lining the vaginal walls

3. Minor secretions of the Bartholin glands

4. Erythema of the genitalia

5. Lengthening, ballooning, and involuntary spasms of the vagina

6. Lifting of the uterus into the false pelvis

7. Descending of the uterus with G-spot stimulation

8. Swelling of the breasts

9. Nipple erections

10. Reddening of the neck and chest of some women

11. Increase in heart rate, blood pressure, and respiration

12. Milky, prostatic-like fluid from the paraurethral gland either emitted out of urethra or present in the bladder or urethra poststimulation 


\section{Table 20.2. Physical reactions during orgasm.}

1. 0.8 second contractions of the vaginal canal

2. Possible contractions of the uterus, rectum, urethra, perineum, and lower abdomen

3. Milky, prostatic-like fluid from the paraurethral gland either ejaculated out of urethra or present in the bladder or urethra poststimulation

4. Heart rate increases to $110-180$ beats per minute

5. Systolic blood pressure increases $30-80 \mathrm{~mm} \mathrm{Hg}$

6. Diastolic blood pressure increases $20-40 \mathrm{~mm} \mathrm{Hg}$

7. Respiratory rate increases up to 40 breaths per minute

8. Muscular tension (different from anxious tension) throughout the whole body

women with strong vaginal and uterine muscle strength will ejaculate the milky prostatic-type fluid that is already present in the arousal phase, from the urethra during orgasm. When not ejaculated externally, this fluid can be found in the bladder poststimulation. The clitoris is probably the most reliable source of stimulation for female orgasm, although some women report a preference and facility for orgasms mediated primarily by vaginal or cervical stimulation. Even women with complete spinal cord injuries have reported laboratory-documented orgasms. The G-spot (an area felt through the anterior wall of the vagina, halfway between the back of the pubic symphysis and cervix, along the urethra) is a common site of intravaginal orgasmic stimulation. If a patient is unsure as to whether she has ever experienced orgasm, preorgasmic sensations may be described as a wave of pleasure that extends over her pelvic area much like the sensation of going over a roller coaster hill followed by the sensation of vaginal contractions and then a feeling of satisfaction. Intense orgasm can be compared to the sudden pleasure and intensity of a sneeze, usually accompanied by a throbbing sensation in the vagina. The vaginal sensations of less intense orgasms feel more like a ripple than an intense series of contractions.

Table 20.2 lists the typical physiologic reactions during the orgasm phase. Orgasm contractions usually last from 3-5 seconds and will be followed either by a sense of physiologic satisfaction (especially in the case of an intense orgasm) or by an urge to continue the stimulation (possibly resulting in more orgasms). When orgasm occurs in a very positive context, the woman often has subjective feelings of satisfaction and/or emotional bonding toward her partner. However, these positive feelings can also result without experiencing the orgasm phase of sexual response, a phenomenon that is difficult for many men to relate to. 
After orgasm, all of the physiologic changes return to the unstimulated state in most women. This is called the resolution phase. A few women will retain the vasocongestion, sometimes resulting in an uncomfortable condition called pelvic congestion syndrome or persistent sexual arousal syndrome.

\section{Establishing the Diagnosis: Sexual Dysfunctions and Problems}

This section lists the definitions, etiologies, and treatment of sexual dysfunctions and problems. The information on pharmacologic and medical causes of dysfunctions cannot be considered all-inclusive, because all conditions and medications listed have not been supported by controlled research, especially in women. Many of the conditions and drugs listed have been supported by research, while others are included because of clinical experience that is consistent with mechanisms of action known to influence sexual response. However, medication effects vary across individuals, with higher dosage levels and greater length of use increasing the incidence of sexual side effects. The clinician is encouraged to refer to the two sexual pharmacology compendia in the reference section to obtain information on the sexual effects of specific drugs as well as preferred alternatives within classes of drugs.

\section{Definitions and Physical Etiologies}

The sexual dysfunctions of female patients are (1) hypoactive sexual desire disorder; (2) arousal disorders; (3) orgasmic disorders; (4) dyspareunia and noncoital sexual pain disorders; (5) vaginismus; and (6) sexual aversion. Other sexual problems that might be encountered include (1) sexual drive discrepancy between the patient and her partner, (2) concern over types of sexual activities, and (3) sexually compulsive behavior in the patient or her partner. In addition, heterosexual patients may seek advice about sexual dysfunction in their male partners, including (1) hypoactive sexual desire disorder, (2) erectile dysfunction, (3) premature ejaculation, and (4) delayed or absent ejaculation.

\section{Hypoactive Sexual Desire Disorder}

This disorder is characterized by a persistent absence or near absence of sexual drive and receptivity that cause personal and/or interpersonal distress. In the general population, about one-third of women reported low sexual drive in the immediate past year of their lives. The range of normality is affected by the patient's age, length and quality of sexual and emotional relationship with her current partner, and her health status. In population-based studies, most women aged 18-29 years engage in partnered sex between two times a week and a few times a month. In contrast, almost a third of women aged 50-59 years claim to be sexually inactive with partners, while $57 \%$ engage in partnered sex a few times a month to a few times a year. Of those who are sexually active 
with partners, the average frequency of sex ranges from seven times a month for young women to four times a month for women in their fifties.

Potential physical causes of low sex drive are listed in Table 20.3. Androgen insufficiency has become increasingly recognized as an important cause of this disorder, and is present in women following oophorectomy or ovarian shutdown from medications or radiation, the binding of free testosterone from oral estrogen medications, hypothyroidism, hypopituitarism, and adrenal insufficiency. Table 20.4 lists psychotropic drugs, Table 20.5 outlines antihypertensives, and Table 20.6 provides information on miscellaneous drugs that have been shown to lower sex drive in some patients.

\section{Sexual Arousal Disorders}

One type of sexual arousal disorder is the persistent or recurrent inability to attain or maintain sufficient sexual excitement as noted by a lack of genital vasocongestion, erotic genital sensitivity, and vaginal lubrication. Another type involves a failure to mentally perceive sexual stimulation as arousing or pleasurable despite adequate physiologic indications. In a study of the general population, around $20 \%$ of women complained either of unpleasurable sex or difficulty with lubrication. Vaginal lubrication and elasticity are highly dependent on estrogen. Likewise, lubrication is dependent indirectly on cholinergic transmitter activity. External genital vasocongestion is also dependent on nonadrenergic/ noncholinergic neurotransmitters like nitric oxide synthetase and polypeptides, such as vasoactive intestinal peptide. Therefore estrogen levels and a review of medications that might interfere with transmitter action are warranted. Also, the conditions or medications that affect sexual desire or orgasmic capacity can affect arousal through a negative feedback loop. In other words, if a woman does not desire to be sexually active because of a testosterone deficiency, she may comply with her partner's initiations but fail to lubricate because of disinterest. There are drugs and conditions believed to inhibit arousal directly and they are listed in Tables 20.7 and 20.8, respectively.

A third type of arousal disorder is persistent sexual arousal syndrome or "PSAS." Prevalence statistics are not available for this disorder. In PSAS, the physiologic responses characteristic of sexual arousal mentioned above may persist for hours or days, and are not necessarily triggered by sexual stimulation or resolved by orgasm. Most, but not all women complaining of this disorder find the symptoms intrusive, worrisome, and sometimes depressing. The research into this syndrome is so new that much is not known about the cause or treatment. All treatments reported to date have failed, but occasionally a patient's symptoms will remit spontaneously.

\section{Orgasmic Disorders}

Orgasm dysfunction occurs when a woman fails to reach that peak level of pleasurable release accompanied by vaginal contractions after sufficient genital stimulation. She is considered dysfunctional only if the condition is persistent and causes her distress. Over $20 \%$ of women in the general population claimed 
Table 20.3. Physical causes of low sex drive.

1. Causes of hormonal imbalances or deficiencies or excesses (involving estrogen, androgens, prolactin)

a. Surgeries-oophorectomy, hypophysectomy, adrenalectomy

b. Chemotherapy that destroys ovarian function

c. Chronic depression or stress

d. Renal dialysis

e. Pituitary tumors-especially craniopharyngiomas and prolactinomas

f. Hypopituitarism

g. Oral contraceptives

h. Alcohol and drug abuse

i. Opiate abuse

j. Oral estrogen replacement therapy

k. Deficiencies of adrenal androgens-dehydroepiandrosterone (DHEA), DHEA-S, androstenedione

1. Rheumatoid arthritis

m. Systemic lupus erythematosis

n. Hypothyroidism

o. Androgen insensitivity syndrome

p. Antiandrogen medications (e.g., for endometriosis)

2. Any disease process or injury that affects the sex centers of the brain

a. Epilepsy-all forms but especially right-sided temporal lobe epilepsy in women

3. Illnesses that affect a woman's general feeling of well-being or her ability to respond sexually
a. Cancer
b. Anemia
c. Diabetes
d. Addison's disease
e. Chronic active hepatitis
f. Chronic renal failure
g. Cirrhosis
h. Cardiomyopathy and other cardiovascular conditions
i. Cushing's syndrome
j. Hemochromatosis
k. Kallman's syndrome
1. Myotonic dystrophy
m. Parkinson's disease
n. Tuberculosis 
Table 20.3. Continued.

o. Chronic obstructive pulmonary disease

p. Chronic pain syndrome

q. Hypertension-treated and untreated

4. Conditions that are often associated with lowered drive

a. Pregnancy and postpartum

b. Perimenopause and menopause

c. Infertility

d. Alcoholism

5. Surgery

a. Any disruption to the nervous or vascular supply to the genitalia can indirectly lead to low sex drive because of the loss of pleasurable feedback to the brain (i.e., colon and rectal surgery for cancer)

b. Any surgery that disrupts the sex centers of the brain

6. Injury-any injury that disrupts the nervous and vascular supply to the pelvis or sex centers of the brain

difficulty with orgasm in the prior year, whereas only $4 \%$ claimed to have never orgasmed. A woman is considered dysfunctional if she fails to achieve orgasm under any circumstance, if her orgasms come so infrequently that sex is disappointing to her, or if orgasm requires such prolonged stimulation that sex becomes a chore. Although many women can learn to reach orgasm with sexual intercourse, failure to reach orgasm regularly with penetration is common and

\section{Table 20.4. Psychotropic drugs that can lower sex drive.}

1. Sedatives/hypnotics

2. Antianxiety agents

3. Antidepressants

a. Tricyclic antidepressants

b. Monoamine oxidase inhibitors

c. Selective serotonin reuptake inhibitors

d. Lithium

4. Antipsychotics/neuroleptics

5. Stimulants/anorectics

Source: Adapted with permission from Crenshaw T, Goldberg J. Sexual Pharmacology: Drugs That Affect Sexual Function. New York: Norton; 1966. 
Table 20.5. Antihypertensives that may lower sex drive.

1. Diuretics

2. Reserpine

3. Methyldopa

4. Guanethidine (Ismelin)

5. $\beta$-Blockers

6. $\alpha_{2}$-Agonists

7. Calcium channel blockers-unlikely unless secondary to depression

Source: Adapted with permission from Crenshaw T, Goldberg J. Sexual Pharmacology: Drugs That Affect Sexual Function. New York: Norton; 1966.

Table 20.6. Miscellaneous medications and treatments that can lower sex drive.

1. Anticonvulsants

2. Anticancer drugs

3. Cardiac drugs

4. Hemodialysis

5. Ophthalmic solutions

Source: Adapted with permission from Crenshaw T, Goldberg J. Sexual Pharmacology: Drugs That Affect Sexual Function. New York: Norton; 1966.

Table 20.7. Drugs known to inhibit vaginal lubrication.

1. Tricyclic antidepressants

2. Anticholinergics, such as those used for asthma

3. Tamoxifen

4. Chemotherapy

5. Monophasic oral contraceptives

6. Any drug that lowers available estrogen

7. Antihistamines 
Table 20.8. Conditions known to inhibit vaginal lubrication.

1. Perimenopause

2. Menopause

3. Vaginitis

4. Expansively growing pituitary adenoma

5. Postpartum-nursing

6. Multiple sclerosis

appears to be normal. However, coital orgasmic capacity is correlated with very strong pubococcygeal muscles. Medical conditions that might cause orgasm dysfunction are listed in Table 20.9 and drugs that can block the orgasmic reflex are listed in Table 20.10.

Table 20.9. Medical conditions that can cause orgasmic dysfunction.

1. Neuropathy

a. Multiple sclerosis

b. Amyotrophic lateral sclerosis

c. Diabetic neuropathy

d. Spinal cord tumors

e. Spinal cord trauma

2. Endocrine disorders
a. Addison's disease
b. Cushing's syndrome
c. Hypothyroidism
d. Hyperthyroidism
e. Hypopituitarism
f. Expansively growing pituitary adenoma
g. Diabetes mellitus
h. Androgen insufficiency

3. Epilepsy

4. Gynecologic factors
a. Surgery that disrupts the pelvic nerve supply
b. Any vaginal condition that causes pain upon stimulation
c. Any chronic illness can indirectly lower orgasmic capacity through malaise, chronic fatigue, or chronic pain


Table 20.10. Drugs that can inhibit orgasm.

1. Antidepressants-tricyclics, monoamine oxidase inhibitors, selective serotonin reuptake inhibitors

2. Antipsychotics/neuroleptics

3. Stimulants-anorectics

4. Tamoxifen

5. Sedatives/hypnotics

Source: Adapted with permission from Crenshaw T, Goldberg J. Sexual Pharmacology: Drugs That Affect Sexual Function. New York: Norton; 1966.

\section{Dyspareunia and Noncoital Sexual Pain}

Dyspareunia (coital pain), which occurs in about $15 \%$ of women in the general population, and noncoital sexual pain can be the result of a variety of physical sources. Types of pain range from itching, burning, or friction of the mucosa, pinpoint sharp pain upon light touch, tightness, and pain upon deep penetration. Even psychological sources can eventually lead to a physiologic cause. For example, failure to arouse mentally can prevent vaginal lubrication causing friction, and fear of pain may cause the vaginal muscles to tighten, making the canal too small to accommodate the penis comfortably. Table 20.11 lists conditions that may cause dyspareunia.

\section{Vaginismus}

Vaginismus is an involuntary contraction of the perivaginal musculature making vaginal penetration impossible, or nearly impossible without significant discomfort or pain. Its prevalence is unclear due to the lack of sampling in populationbased studies. However, clinical experience suggests that this problem is being presented at increasing rates to health care providers due to the impact of vulvodynia. Vaginismus is often caused by a prior history of dyspareunia for reasons listed in Table 20.11, by fear of painful intercourse learned from folklore, or by a history of sexual abuse. This condition can be worsened by the gynecologist who is too eager to complete a pelvic examination, despite the patient's warnings of prior painful penetrations or painful protestations during the pelvic examination. Women with vaginismus sometimes retain normal sex drive and the ability to achieve orgasm by noncoital methods.

\section{Sexual Aversion/Phobia}

Sexual aversion is the negative or phobic reaction to sexual activity or to the anticipation of sexual activity. Sometimes aversion to a particular sexual activity, 
like fellatio, or to a sexual part, like the penis, makes the woman avoid all sexual activity. When aversion is of phobic proportions, physical reactions are those of panic disorder: overwhelming anxiety, sweating, nausea, vomiting, diarrhea, or palpitations. Once sexual activity begins, some women proceed through the sexual response cycle. A previous history of dyspareunia and low testosterone could be physical precursors to this condition, though most causes are believed to be psychological.

\section{Table 20.11. Physical conditions that can cause dyspareunia.}

1. Rigid hymen

2. Painful hymeneal tags

3. Endometriosis

4. Pelvic inflammatory disease

5. Atrophic vaginitis

6. Relaxation or laceration of broad ligaments supporting the uterus

7. Pelvic tumors

8. Poor episiotomy repair

9. Vulvodynia

10. Interstitial cystitis

11. Ectopic ureter

12. Vaginal stenosis

13. Urethral caruncle

14. Hemorrhoids

15. Vaginal disease-herpes, candidiasis, human papilloma virus, etc.

16. Allergic reaction to rubber products used in condoms and diaphragms

17. Allergic reaction to contraceptive chemicals

18. Allergic reaction to semen

19. Repeated douching upsetting the vaginal $\mathrm{pH}$ value

20. Pelvic surgery

21. Estrogen deficiency during the postpartum nursing phase

22. Forced intercourse causing tearing of the vaginal mucosa

23. Levator ani myalgia from overuse of Kegel exercises

24. Urethritis

25. Radiation therapy

26. Pelvic adhesions

27. Adnexal pathology (i.e., ovarian cyst)

28. Symptomatic uterine retroversion

29. Pelvic floor disorders 


\section{Other Sexual Problems}

Sexual drive differences between partners constitute one of the most common sexual concerns presented to sex therapists. It is not necessarily accompanied by sexual dysfunction in either partner. When drive differences cause significant problems, it is often because one partner believes that his or her drive is the norm and the other partner's drive is abnormal. Physicians can educate their patients about the wide range of normal sexual frequencies and discuss options for compromise.

Another sexual problem commonly encountered is the discrepancy between a woman and her partner over the type of sexual activity they enjoy. The inclusion of oral sex is a common source of disagreement between partners. These discrepancies, if left unresolved, can lead to sexual dysfunction. The physician can assess whether the patient needs accurate information about the normality of a given activity, how to engage in sexual practices effectively, or how to prevent injury or disease. Oral sex participants should know that many sexually transmitted diseases can be transmitted orally and condoms or dental dams should be used when there is a risk of disease. Additionally, there is a statistically significant, positive association between oral sex and repeated vulvovaginal candidiasis.

Likewise, anal sex recipients should be encouraged to lubricate and gradually dilate their anal openings before penetration with a penis or dildo to prevent tears of the mucosa. Frequent anal eroticism is associated with anorectal pain, ulcers or fissures, rectal prolapse or leakage, and hemorrhoids. Condoms should be used to prevent transmission of sexually transmitted diseases (STDs). There should be no penetration of the vagina with anything that has just been in the rectum because normal bacterial flora in the rectum will cause infection.

\section{Sexual Addiction/Compulsion}

The physician may encounter sexually compulsive behavior in the patient or her partner. This problem is defined as a pathologic need for sexual activity that gradually replaces the desire to have healthy relationships and activities. And while compulsive sexual activity can be secondary to other compulsive, addictive, mood, or posttraumatic stress disorders, it may also be a primary comorbid condition. Clues to sexual compulsivity that may present in the gynecologist's office include genital injury from compulsive masturbation, repeated STDs or unplanned pregnancies, a history of multiple sexual partners in a short period of time, or a demanding attitude about frequency or activity. This patient may also be seductive with her physician.

More often, the patient is the victim of her partner's sexual compulsions. In this situation she may present with genital injury, STDs, a loss of sex drive due to the excessive demands of her partner, or a complaint of infrequent sex due to the partner acting compulsively outside of the relationship. Use of Internet pornography has become an increasing complaint of sex 
therapy patients, with research showing some participants spending over 20 hours a week in such activities-neglecting family, job, or the need for adequate sleep. Patients with simply high sex drives, or practices that are not part of the cultural norm, can be misdiagnosed as compulsive by conservative partners or clinicians. Such diagnoses should be left to sexual specialists. Furthermore, true sexual compulsion is a very complex problem to treat and almost always warrants a referral to a sex therapist.

\section{Male Sexual Dysfunction}

Sexual dysfunction in the heterosexual patient's partner may lead to her own sexual dysfunctions. A patient may also seek guidance for the partner's dysfunction. It is important for the gynecologist to know how to diagnose and refer these patients. (Lesbian partner's dysfunctions can have similar effects on your patient and likewise need to be addressed.) Male sexual dysfunctions are listed in Table 20.12.

\section{Hypoactive Sexual Desire Disorder}

Hypoactive sexual desire disorder in a male is essentially the same phenomenon as in women in definition and causes (see related tables). One main difference is in the role of estrogen, which, when excessive in the male, lowers his drive. Some diseases, such as alcoholism, result in a relative increase of estrogen in a man's body because of the pathologic state of the liver. In other cases, medications may raise his estrogen levels to sexually dysfunctional proportions. A man with a global decline in sex drive should be evaluated by his primary care physician. However, the man whose sex drive has remained intact in general but is turned off only by his current partner can be referred for couples therapy or to a sex therapist.

\section{Erectile Dysfunction}

Erectile dysfunction is a failure to attain an erection during genital stimulation, or failure to maintain an erection until ejaculation or the man's desire to stop. This label also applies to erections lacking adequate rigidity for penetration. The ability to maintain an erection to ejaculation with manual stimulation, or with an alternate partner's stimulation, is a good indicator that there is no

\section{Table 20.12. Common male sexual dysfunctions.}

1. Hypoactive sexual desire disorder

2. Erectile failure

3. Premature ejaculation

4. Delayed or absent ejaculation 
physical cause. Decreasing frequency of morning erections or inability to keep morning erections with body movement may be signs of organic etiology. Referral to a urologist who specializes in sexual dysfunction is the safest way to ensure diagnostic accuracy. As psychogenic erectile dysfunction is more adequately treated if it is addressed sooner, prompt referral to an appropriate specialist is important.

\section{Premature Ejaculation}

Premature ejaculation occurs when a man lacks the ability to exert some control over his ejaculatory latency. Usually this diagnosis is more appropriate when the man ejaculates sooner than several minutes after penetration. The human male would naturally ejaculate within 2 minutes of vaginal thrusting if he were not purposefully delaying this reflex. A patient might complain of the partner's premature ejaculation because she has not reached orgasm with vaginal penetration, even after as much as 10 minutes of thrusting. This patient merely needs to be educated about the relative differences in vaginal and penile sensitivity and the importance of clitoral stimulation and increased foreplay before penetration. Premature ejaculation is rarely a result of medical conditions, and unless the man is complaining of other symptoms indicative of urinary tract infection or prostatitis, referral to a sex therapist is appropriate.

\section{Delayed Ejaculation}

Delayed ejaculation occurs when a man or his partner complains of his inability to ejaculate after sufficient stimulation to the penis. The inability to ejaculate within 5-10 minutes (when a man is not intentionally suppressing this reflex to please his partner) can be considered dysfunctional. As with female orgasmic dysfunction this definition is subjectively defined by the couple, and especially the man's subjective sense of a failure to progress to orgasm. When the man has no difficulty ejaculating with noncoital stimulation, the problem is assumed to be psychogenic and referral to a sex therapist is appropriate. However, diseases such as diabetes and medications such as selective serotonin reuptake inhibitors (antidepressants) are often associated with this symptom and referral to an internist or urologist is recommended.

\section{Psychological Causes of Sexual Dysfunction}

Table 20.13 lists the five main categories of psychological causes of sexual dysfunction: immediate, relational, psychiatric, historical, and societal.

\section{Immediate Causes}

Immediate causes of sexual dysfunction are those that occur during the lovemaking process and include the following.

\section{Spectatoring}

This phenomenon occurs when a person mentally stands outside herself and watches during sexual activity. As she watches, she will often internally criticize 


\section{Table 20.13. Psychological causes of sexual dysfunction.}

1. Immediate-spectatoring, performance anxiety, need to please, negative focus, ineffective stimulation, lack of attraction, distracting fantasies/ thoughts

2. Relational-anger, power struggle, infidelity, mistrust, lack of emotional intimacy, fear of pregnancy, divergent preferences, sexual addiction/compulsion

3. Psychiatric-depression, anxiety, posttraumatic stress disorder, some personality disorders

4. Historical-religious repression, sexual trauma, negative parental input

5. Societal-myths, media, double standard

herself whether or not that evaluation has any basis in reality. These mental processes lower her mental pleasure and send inhibitory messages to the pelvic nerves, thus damping her sexual reflexes.

\section{Performance Anxiety}

This occurs when a woman has certain expectations of the "right" way to respond sexually and does not live up to her own expectations.

\section{Excessive Need to Please}

A woman may have trouble responding if she is focused so much on her partner's pleasure that she does not attend to the type of stimulations or conditions she needs to be aroused.

\section{Focusing on Negative Aspects}

Sometimes a woman focuses on certain negative characteristics of her lover during or just before the sexual experience-body type, manner of dress, etc. Although perhaps justifiable, this may also stem from being a perfectionist and thus unable to focus on the total love-making experience.

\section{Lack of Proper Stimulation}

Although commonalities exist, each woman is unique in her preferences. Furthermore, the same stimulation may produce opposite reactions in the same woman at different times. Thus, it is very important that the woman be free to communicate her sexual preferences to her partner. The clinician should urge the patient to be a good communicator.

\section{Lack of Physical/Chemical Attraction to Partner}

Research demonstrates that women are less likely to require sexual attraction when selecting their committed partners versus their dates. After the newness of a relationship wears off, a woman may find sex with her partner of decreasing appeal. An often overlooked factor in women's arousal is the bodily scent of 
her partner. Therefore, a woman turned off by her partner's scent will be negatively affected by a common precursor to women's sexual receptivity-the physically affectionate touching that precedes sexual interaction. Such partners should pay strict attention to their hygiene to minimize this impact.

\section{Unwanted Fantasies/Thoughts}

Sometimes disruptive fantasies and thoughts occur during sex. They may include images of prior sexual abuse causing anxiety and disgust, images of other desirable sexual partners or taboo, but arousing sexual activities inducing guilt, or simply images or thoughts of which chores need to get done.

\section{Relational Causes}

Relational causes of sexual dysfunction, which occur with one's sexual partner, are characterized by the following.

Displaced Anger. Unresolved anger often affects the ability and desire to respond to one's partner sexually.

Power Struggle. Some individuals withhold sex to punish or engage in sex to manipulate their partner, in response to a lack of power or agreement in other aspects of the relationship.

Infidelity. If the patient has had an affair, her drive toward her committed partner may decrease out of guilt, a sense of loyalty to her other lover, or fear of infecting her committed partner with an STD. Likewise, if her partner has been unfaithful, her drive may decrease as a result of a feeling of betrayal, depression, anger, or fear of contracting a disease.

Mistrust. In addition to the mistrust that results from an affair, women's drives may be decreased by their inability to trust their partners with the intimate physical and verbal reactions that come from uninhibited sex. Similarly, they may refuse sex because they are not certain that the relationship will last.

Lack of Emotional Intimacy. Women tend to respond best when they feel emotionally close to their partners. Poor communication patterns and lack of personal differentiation can prevent emotional intimacy from developing in a couple.

Fear of Pregnancy. For a woman to respond best during sex, she must be uninhibited and free of fears. The woman who is not ready to risk a pregnancy may keep herself "in check" so that she will not have intercourse or forget to use contraception.

\section{Divergent Preferences}

Some women avoid sex because of the fear of being pressured into unappealing sexual activity (such as anal sex). Others may not respond well because their partners do not want to touch them the way they most prefer. Remind the 
patient that a healthy partner appreciates not having to guess, is aroused by his or her partner's forthrightness, and is willing to find sexual activities that appeal to both.

\section{Sexual Compulsivity}

Compulsive tendencies may cause a decrease in responsiveness as a result of oversatiation. Likewise, sexual activity may not be particularly pleasurable because the goal may be to numb negative emotions rather than to express sexual urges. The woman with a sexually compulsive partner may become less responsive because of the pressure to be sexual regardless of her feelings or needs. Conversely, some compulsive partners engage in sex elsewhere, leaving the woman to complain of infrequent sex.

\section{Psychiatric Disorders}

Psychiatric disorders can produce sexual problems. The most frequent associations are with depression, anxiety disorders, posttraumatic stress syndrome, and alcoholism or drug addiction. Other common psychiatric disorders, such as personality disorders, can be related to sexual dysfunction, but not necessarily.

\section{Historical Inputs}

Historical inputs to sexual dysfunction, including events in the individual's upbringing that have negatively affected current sexual attitudes, may occur through the following ways.

\section{Religion}

The Judeo-Christian ethic has traditionally been sexually repressive, assigning negative attitudes to sexual pleasure. While this message has changed in some religions, the current trend of sexual conservatism has resulted in mixed messages, leaving young newlyweds with sexual anxiety and guilt, aging couples with the belief that sex solely for pleasure is inappropriate, and homosexual individuals struggling to function within heterosexual marriages.

\section{Sexual Trauma}

An estimated one-third of women have experienced sexual abuse in their past. This is most often associated with subsequent sexual dysfunction, but compulsive and irresponsible sexual activity can also result. Adolescent girls who have been abused are more likely to attempt to or to become pregnant and to initiate sex at a younger age than their nonabused peers.

\section{Parental Influences}

Parents convey sexual attitudes to their children whether they refer to sex directly or not. By not educating children positively about the sexual aspects of their bodies and feelings, or by conveying only the consequences of sex, parents inadvertently convey a negative or shameful attitude about sex and sexual body parts. 


\section{Societal Causes}

Societal causes, which are those generalized beliefs and behaviors within a culture that affect women's sexual self-image and self-esteem, are conveyed through the following.

Myths. Our society has a history of withholding sexual information, and myths develop out of this ignorance. A very common myth is that women should find sexual intercourse as physically stimulating as men. Another is that what stimulates one person will automatically stimulate that person's partner.

Media. Use of beautiful, slender models in media advertising has resulted in a negative body image in some women. These women tend to focus on their perceived bodily imperfections during sex rather than on their pleasure.

Double Standard. The double standard still exists, causing women confusion about claiming their sexuality. Sexually assertive men may be revered, whereas the same behavior in women may be seen as "loose." This double standard extends to some medical sexual studies when it ignores the research on the complexity of female sexual function and satisfaction, and how often the sexual responses of females are attained differently from those of males.

\section{Management of Sexual Problems Sexual History}

Taking a thorough sexual history of a patient is the key to accurate diagnosis and treatment. Three types of histories, listed in Table 20.14, are appropriate in an obstetric-gynecology practice: one occurs within a routine review of systems during an annual examination or request for a physical; the second occurs when the physician is consulted about physical symptoms that may be related to sexual activity; and the third occurs when a patient specifically discloses a sexual dysfunction and asks for help. A sex history can be covered in several minutes.

Although a high percentage of patients (85-91\%) believe it is appropriate for physicians to take sexual histories, studies show that physicians are generally reluctant to initiate questions concerning sex with their patients for fear of seeming inappropriate, because they feel unskilled, or because they believe they do not have time to cover this area. Other physicians are reluctant

\section{Table 20.14. Types of sexual histories.}

1. Routine review of systems

2. Secondary to pelvic symptomatology

3. Sexual dysfunction 
to bring up sex because of their own embarrassment or lack of skill in discussing sex or because they do not want to make their patients uncomfortable. Because research shows that more than half of women admit to sexual problems when specifically asked, and because STDs can have far-reaching health consequences for women, it is imperative that clinicians initiate these discussions.

Effective sexual history taking requires more sensitivity than other reviews. A patient is more likely to be honest if she believes that her physician is interested, nonjudgmental, and comfortable with the topic. Physicians who are uncomfortable are encouraged to seek guidance from an experienced sex therapist and/or attend a "Sexual Attitude Reassessment" seminar (for a list of SARs call The American Association of Sex Educators, Counselors, and Therapists-804-752-0026). Questions should be worded in a way that makes no assumptions about sexual orientation or monogamy.

\section{Sexual History Taking During a Routine Examination}

The form of sexual history taking shown in Table 20.15 is used as a screening device for sexual activities that may affect a woman's health or sexual satisfaction. In addition, it can provide a baseline of functioning that can be used for comparison in later years to more effectively assess the impact of gynecologic surgeries, medication, menopause, or pregnancy on the patient's sexuality.

This sexual data gathering seems to flow best at the end of a menstrual history. Using the same matter of fact tone of voice and pacing, proceed with the questions in Table 20.15. If you are fearful of being misunderstood, begin with something like this: "Ms. Jones, I'd like to ask you some questions about your sexual functioning if you don't mind. Sexual activity can affect your health and your health can affect your sexual activity. I want to make sure I don't miss anything important. I am not here to judge you, so please feel free to be candid with me."

Some of the questions in Table 20.15 are best asked the first time a patient is seen. For ongoing patients, use judgment to modify questions based on what is already known about the patient. For example, a long-term patient whose previous history shows she is monogamous and heterosexual might be insulted by questions that suggest she is not. It is best to review the sexual functioning questions in terms of whether the patient has developed any new symptoms or concerns since the last visit. Try to assess the patient's level of sophistication with vocabulary and modify questions using the terms with which she seems comfortable. It may be necessary to define some terms, especially if the patient seems confused. Likewise, begin questions with "how often do you" or "when you." Phrases such as "have you ever" suggest that such a practice is unusual and is likely to elicit a false-negative reply.

If the patient seems unusually uncomfortable with sexual history taking, stop and note her discomfort and ask her if she would like to talk about it. She may reveal that she experienced sexual abuse as a child and has kept this a secret. Be sure to stop the history taking and show appropriate 
Table 20.15. Questions for a detailed sexual history.

Are you currently sexually active? Have you ever been?

Are your partners men, women, or both?

How many partners have you had in the past month? Six months? Lifetime?

How satisfied with your (and/or your partner's sexual functioning are you? Has there been any change in your (or your partner's) sexual desire or the frequency of sexual activity?

Do you have, or have your ever had, any risk factors for HIV? (List blood transfusions, needlestick injuries, IV drug use, STDs, partners who may have placed you at risk.)

Have you ever had any sexually related diseases?

Have you ever been tested for HIV? Would you like to be?

What do you do to protect yourself from contracting HIV?

What method do you use for contraception?

Are you trying to become pregnant (or father a child)?

Do you participate in oral sex? Anal sex?

Do you or your partner(s) use any particular devices or substances to enhance your sexual pleasure?

Do you ever have pain with intercourse?

Women: Do you have any difficulty achieving orgasm?

Men: Do you have any difficulty obtaining and maintaining an erection? Difficulty with ejaculation?

Do you have any questions or concerns about your sexual functioning? Is there anything about your (or your partner's) sexual activity (as individuals or as a couple) that you would like to change?

$\mathrm{HIV}=$ human immunodeficiency virus; IV = intravenous; STDs = sexually transmitted diseases.

Source: From "The Proactive Sexual Health History" Nusbaum M, Hamilton C, (authors) American Family Physician Vol. 66(9): November 1, 2002.

concern. The patient may feel she is taking a major risk in revealing this information. Another patient may be uncertain of the physician's motives in asking sexually explicit questions. If the prologue mentioned above was not used, now is the time to briefly explain that sexual symptoms and dissatisfaction can affect a woman's health and how well she takes care of herself. After explaining this to the uncomfortable patient, always ask her permission to 
continue with the sexual history. If she will not allow it, nonjudgmentally suggest that if she ever wants to discuss her concerns in the future, you are willing to do so. Likewise, offer a referral to a therapist if she reveals a history of abuse or dysfunction. It may take multiple visits for a patient to build enough trust to reveal her sexual concerns. By opening the door each time with sexual questions, it allows her to address these issues when she is ready, without having to openly admit that she withheld information in the past.

Most physicians' concerns about sexual history taking can be alleviated by showing their patients they care about their health and are sensitive to the embarrassment that many have in discussing sex.

\section{Sexual History Taking following Patient Presentation of Vaginal Symptoms}

Parts of the same sexual history used for routine examinations noted in Table 20.15 can be used for the patient with pelvic symptomatology. Just preface these questions differently, such as, "Ms. Johnson, sometimes the symptoms you are having are related to a woman's sexual practices. I'd like to ask you some questions that could help me treat your symptoms more successfully. Please feel free to be honest. I just want to help you get over these symptoms."

\section{Sexual Dysfunction History}

Table 20.16 lists general questions to ask when a patient presents with a sexual dysfunction or when you have uncovered a problem in a routine screening. Note that a patient with no personal or interpersonal distress about her sexual responses needs no treatment unless she specifically wants to improve her functioning. Obtain information on the entire sexual response cycle of the patient and her partner, as one dysfunction may be at the root of another. For example, a patient who presents with low sex drive may have dyspareunia as the causative factor. Assess desire, lubrication/erection, orgasm/ejaculation, presence of sexual pain, and satisfaction regardless of which dysfunction is initially presented. If the patient shows distress in discussing these problems, be sure to make empathic statements such as "this has really bothered you, hasn't it?"

If the patient's complaints include decreased sexual desire, she should always be screened for depression. Table 20.17 lists the appropriate questions. A positive reply in several areas indicates a need for further evaluation by an internist and possibly a referral to a psychiatrist or experienced nonmedical therapist. Reassure the patient that she will get a thorough assessment and appropriate treatment.

\section{Office Management of Sexual Dysfunction}

It should be noted that diagnostic tools for identifying organic causes of female sexual dysfunction are greatly underresearched and lag far behind those for 
male sexual dysfunction. Vaginal photoplethysmography is of some benefit and other measures of clitoral and labial temperature and blood flow are being developed. Well-researched neurologic tools for genital sensitivity are needed as well as greater understanding of hormonal and other neurochemical mediators. Furthermore, current testosterone assays for normal female levels are unreliable at the lower ranges and laboratory ranges are not correlated with good sexual functioning. Therefore the recommendations given below may change greatly over the next few years.

\section{Table 20.16. Sexual dysfunction history.}

1. Describe your symptoms. (Be very explicit about this, especially with dyspareunia)

a. Dyspareunia. Where do you feel the pain-general, localized, with deep penetration, upon entry? Describe the pain-sharp, raw, burning, too small for anything to enter?

2. When did this problem begin?

3. Describe the circumstances at the time of onset
a. With masturbation or with which partner
b. Patient's mental health-depression, anxiety, stress, etc.
c. Medical problems or surgeries
d. Intoxication with mood-altering drugs by patient or her partner (i.e., alcohol, marijuana)
e. Nature of relationship to partner
f. Use of prescription drugs, herbal medicines, or over-the-counter drugs
g. Pregnancy, infertility treatments, menopause/perimenopause
h. Concurrence with sexual pain

4. Does this problem change in severity or frequency? If so, what makes it better or worse?

5. Do you or your partner have difficulty in other sexual responsesdesire, lubrication/erection, or orgasm/ejaculation?

6. What do you believe caused this problem? (for example, differences in sexual style, frequency, ineffective stimulation, coincidental with medication or illness onset, history of sexual trauma)

7. Have you sought other professional help for this? If so, what was done? Results? May I consult with that professional?

8. What treatments have you tried on your own?

9. Do you have any ideas about what should be done to help you overcome this symptom? 


\section{Table 20.17. Depression screening.}

1. Do you feel depressed? (A negative reply does not necessarily rule out depression)

2. Is your sleep disrupted in any way, such as difficulty getting to sleep within 10-15 minutes, waking during the night with difficulty getting back to sleep easily, awakening 1-2 hours earlier than you need or want to?

3. Has your appetite greatly increased or decreased?

4. Has your weight fluctuated more than 10 pounds without effort on your part (not due to smoking cessation)?

5. Do you experience constipation? Diarrhea?

6. Has your drive for sex been lowered under all circumstances?

7. Has your energy decreased a lot?

8. Has your concentration decreased a lot?

9. Are you feeling excessively guilty about something?

10. Do you feel inadequate or like a failure?

11. Do you have persistent thoughts of death or suicide? (A positive reply to this question warrants immediate referral to a psychiatrist.)

\section{Low Sex Drive}

If the history and physical examination suggest it, laboratory studies should be done to screen out physical causes of low sex drive, especially hypothyroidism and hypopituitarism. Androgen deficiency is emerging as a likely medical cause of this disorder, especially if the patient is well estrogenized. A woman with insufficient androgens will give a history of persistent absent or almost absent sexual drive for partnered sex, masturbation, and erotica, and few if any sexual dreams. Additionally, she may complain of a persistent unexplained fatigue, lowered mood, and decreased sexual receptivity, orgasm, and pleasure. Recommended laboratory screening for low sex drive is presented in Table 20.18. There are no large-scale studies documenting minimum androgen levels needed for adequate sexual functioning, however, when androgen insufficiency needs to be ruled out. The Princeton Conference on Androgen Insufficiency in Women (2002) provides us with our current guidelines listed in Tables 20.19 and 20.20. (Androgen insufficiency should be suspected when women complain of the symptoms listed in Table 20.19. The diagnosis of androgen insufficiency can be made using the guidelines in Table 20.20.) There are no large-scale studies to determine androgen levels adequate for sexual function; however, Table 20.21 contains a range of levels correlated with good sexual functioning in a small sample of reproductive aged women. Androgen levels 


\section{Table 20.18. Suggested laboratory screening for low sex drive.}

1. Estradiol or follicle-stimulating hormone (if symptoms of deficiency)

2. Serum testosterone

3. Free testosterone-equilibrium dialysis preferred or calculated free $T$

4. Dehydroepiandrosterone sulfate (DHEAS)

5. Thyroid profile

6. Prolactin (if symptoms warrant)

should be obtained in the morning, but there is lack of consensus as to whether they should be drawn in the follicular phase, when androgens are lowest, or in the midcycle, when testosterone peaks at ovulation.

Treatment for most physical causes of low sex drive listed in the tables is well documented. However, treatment recommendations for androgen insufficiency are just emerging and thus it will be discussed in detail here. It has been suggested that because testosterone assays are not sensitive at lower levels of female normal ranges, a free testosterone level in the lower quartile of the normal range accompanied by symptoms of testosterone deficiency warrants a trial of replacement therapy. Ironically, because oral contraceptives lower free testosterone, replacing them with nonhormonal methods may be all that is needed in some women. Likewise, women on oral forms of estrogen (replacement) therapy should switch to a transdermal approach, which does not bind free testosterone except at high doses. If sexual symptoms persist 6-12 weeks after these medication changes, laboratory tests should be repeated to rule out continued androgen deficiency. Options for androgen replacement therapy are presented in Table 20.22.

Research results indicate that injectable forms of testosterone produce reliably positive sexual drive effects in oophorectomized women and have not

\section{Table 20.19. Symptoms of androgen insufficiency.}

1. Decreased sexual drive, pleasure, genital sensitivity, or orgasm

2. Persistent, unexplained fatigue

3. Low mood

4. Decreased vaginal lubrication even when adequately estrogenized

5. Changes in cognition and memory

6. Decreased muscle strength

7. Bone loss

8. Hot flashes despite adequate estrogen 
Table 20.20. Criteria for diagnosing androgen insufficiency.

1. Clinical symptoms are present

2. Adequate estrogen—regular menstrual periods or estrogen replacement therapy

3. Free testosterone is in the lowest quartile (or lower) for norms based on women aged $20-40$ years

Table 20.21. Androgen laboratory norms ${ }^{\mathrm{a}}$ correlated with positive female sexual function.

Age range

\begin{tabular}{lccc}
\hline Age & $20-29(17)$ & $30-39(23)$ & $40-49(20)$ \\
Total T $(\mathrm{ng} / \mathrm{dl})$ & $45-57$ & $28-40$ & $27-39$ \\
Free T $(\mathrm{pg} / \mathrm{ml})$ & $1.4-1.6$ & $1.0-1.2$ & $0.9-1.1$ \\
DHEA-S $(\mu \mathrm{g} / \mathrm{dl})$ & $177-214$ & $139-171$ & $125-156$ \\
SHBG $(\mathrm{nmol} / \mathrm{liter})$ & $44-59$ & $45-52$ & $47-58$ \\
\hline
\end{tabular}

${ }^{\text {a }}$ Drawn in the morning on days 8-15 for premenopausal women with no sexual dysfunction. T, testosterone; DHEA, dehydroepiandrosterone; SHBG, sex hormone-binding globulin.

Source: Reproduced with permission from Guay A. Androgen values in premenopausal women without sexual dysfunction. Podium \#6. Proceedings of the International Society for the Study of Women's Sexual Health Annual Meeting. 2002.

Table 20.22. Androgen therapy options for women.

1. $\quad 1.25-2.50 \mathrm{mg} /$ day methyltestosterone

2. $75 \mathrm{mg}$ testosterone enanthate IM for 28 days postoophorectomy

3. $200 \mathrm{mg}$ dihydroandrosterone IM for 28 days postoophorectomy

4. $300 \mathrm{mg}$ testosterone patch for a month

5. 50-100 mg dehydroepiandrosterone (DHEA) daily am 
been shown to adversely affect high-density lipoprotein (HDL) and liver toxicity like oral forms. While clinical experience attests to the efficacy of injectable routes of testosterone administration in premenopausal, testosterone-deficient women, there are no published data to support this approach while keeping testosterone levels within physiologic ranges. Once replacement begins, laboratory tests need to be repeated every few months to make sure the patient stays within normal ranges. Though not well supported by controlled research results, methyltestosterone (oral form) dosed at $1.25-2.50 \mathrm{mg} /$ day is effective for some women and is available for hormone therapy (HT) either as Estratest or can be individually compounded. Surgically menopausal women have not demonstrated any hepatotoxicity at dosages of $2.5 \mathrm{mg}$ of methyltestosterone daily, but have demonstrated adverse trends in lipoproteins. Furthermore, research on higher replacement dosages of methyltestosterone (often in men) reports significantly lower HDL cholesterol, liver toxicity, and polycythemia. Therefore, pretreatment laboratory measures of hepatic function, lipid profile, triglycerides, and hematocrit, as listed in Table 20.23 , are indicated with repeat studies in intervals of several months to 1 year once replacement begins.

In controlled studies, dehydroepiandrosterone (DHEA) tablets have been shown to be effective in raising sex drive and responsiveness for pre- and postmenopausal women with documented adrenal insufficiency and for premenopausal women with hypopituitarism. The overall efficacy of DHEA for premenopausal women with androgen insufficiency remains to be clarified. Effective dosages ranged from $30-50 \mathrm{mg} /$ day. However, finding a brand of DHEA with guaranteed potency is difficult since it is unregulated by the Food and Drug Administration (FDA). Both oral forms of androgen replacementDHEA and methyltestosterone-appear to be safe in the short run in the dosages mentioned. Controlled research on testosterone gels in this population so far shows positive sexual effects only when free testosterone was elevated to supraphysiologic ranges.

Anecdotally, a 2\% testosterone cream has also been used with mixed feedback as to its effectiveness for libido problems, possibly because some patients tend to use insufficient doses to bring testosterone into normal ranges. The lack of controlled studies on this route of administration leaves the clinician with only experiential guidelines and repeated laboratory assays

\section{Table 20.23. Laboratory tests prior to and during androgen replacement.}

1. Lipid profile-total, high-density lipoprotein, low-density lipoprotein

2. Hepatic function

3. Triglycerides

4. Hematocrit 
to determine if dosages are producing improved levels of circulating androgens. The same is true for testosterone gels, modified from male dosages that are currently available. However, controlled research does support the efficacy of a testosterone patch in raising several sexual function parameters in oophorectomized women, but this approach currently lacks FDA approval until longer term safety studies can be conducted. This product has excellent shortterm safety results. No matter which route of hormone administration is used, patients will likely need several months-possibly 4-6-of consistent treatment, with regulation of dosages often based on repeated laboratory assays and symptom relief, before positive effects can be seen or androgen replacement therapy can be considered ineffective.

The most common side effects of any form of testosterone replacement therapy include weight gain, increased facial hair, and acne. However, when levels are kept within normal ranges, these side effects are not common and do not require discontinuation of treatment. Lipid profiles may worsen, especially with oral routes, and therefore need to be monitored before administration and at intervals of several months during usage. Dosages can be lowered to obtain a balance between sexually enhancing effects and unwanted side effects.

If desire stays low despite several months of documented normal androgen values, the couple should be referred for sex therapy. Replacement therapy can be continued during the course of psychotherapy to see if the combination produces effective results. However, couples with long-term desire difficulties and androgen insufficiency often benefit from combining sex therapy and androgen replacement therapy at the beginning of treatment, to counteract negative attitudes and ineffective coping strategies.

Reproductive aged women who choose androgen replacement therapy should be informed of the potential masculinizing risks to a fetus and be encouraged to use highly reliable birth control methods. Until long-term studies can document the safety of androgen replacement therapy, women with an already adverse lipoprotein profile, documented atherosclerotic cardiovascular disease, or a marked family history of cardiovascular disease, diabetes, or breast cancer should be informed of the potential for exacerbation of these diseases or their side effects with androgen replacement therapy.

Patients with no discernible medical cause for low sex drive should be referred with their partners to a sex therapist. Sometimes the patient presents with a complaint of low sex drive based only on the discrepancy of drives between partners. When no sexual dysfunction is present, couples should be encouraged to use the following paradigm. If the patient is approached for sex but would resent participation, her partner can be encouraged to masturbate, if that is within the partner's value system. If the patient is neutral, she might propose a sexual encounter where no expectations about outcome would be the ground rule. That way, if she does not become aroused, her partner need not take it personally, but can nonetheless benefit from the stimulation received. Similarly, the sexually neutral patient can offer to stimulate her 
partner without receiving any stimulation in return. Of course, if any of these solutions become the norm, or if both people are not willing to be flexible, a referral for sex therapy is warranted.

For all the sexual dysfunctions, the obstretic-gynecologic clinician can provide immediate help in the form of accurate information, permission to be sexual, or encouragement to communicate the patient's sexual needs to her partner. However, because low drive tends to be a multifactorial problem, treatment often requires a trained sex therapist who may recommend a combination of psychotherapy, couples therapy, bibliotherapy, and at-home sexual exercises. If there is sexual abuse in the patient's history, direct referral to a psychotherapist is indicated.

\section{Arousal Disorder}

Standard applications of vaginally inserted estrogen cream or tablets in those with laboratory documented serum deficiencies of estrogen or who otherwise show signs of vaginal atrophy should be prescribed for those lacking genital vasocongestion and lubrication. If coincident with androgen insufficiency, lack of genital sensitivity can be treated with a topical testosterone cream applied to the vulva, though its effectiveness is yet to be documented with controlled research. Other androgen replacement options mentioned above could be considered as well. In selected cases, oral vasodilators such as Sildenafil have increased genital vasocongestion with sexual stimulation. Clinical trials are under way to determine the effectiveness of Sildenafil and other vasodilators such as L-arginine, yohimbine, apomorphine, and phentolamine. Topical prostaglandin $\mathrm{E}_{1}$ and vasoactive intestinal peptide are also under investigation for arousal disorder.

If physical causes of this disorder have been ruled out, and the patient's sexual attraction for her partner is intact, her problem may be caused simply by a lack of proper stimulation. The patient should be encouraged to communicate the type of touch she wants to her partner. If not already known from prior experience, this can be learned through masturbation or by reading books designed to help women to orgasm. Often she will need emotional connection or a different type of initiation to become receptive, but she has failed to communicate to her partner how that can be established. Women who are unable to follow through with communication or whose arousal disorder is not amenable to simple education and permission giving by their physician will need psychotherapy to address the more complex causes such as relationship problems or sexual trauma.

\section{Orgasm Disorder}

Orgasmic dysfunction may be medical in etiology. Certainly physicians should assess for symptoms indicative of medical causes listed in the tables, for example, unregulated diabetes or diabetic neuropathy, androgen deficiency, and selective serotonin reuptake inhibitor (SSRI) antidepressants. Sexual dysfunction in diabetics may subside once blood sugar levels are brought under control. 
Androgen insufficiency should be treated using the guidelines in the low sex drive section. Preliminary controlled trials show that the testosterone/estrogen patch can significantly improve orgasm and pleasure beyond estrogen replacement alone in surgically menopausal women; however, not all women low in testosterone have difficulties with orgasm. Likewise, Tibolone, a drug with androgenic, estrogenic, and progestogenic activity (not available in the United States) is showing significantly positive sexual effects on orgasm and responsivity in surgically menopausal women compared to estrogen replacement therapy alone. However, its adverse impact on lipid profiles is a concern.

A $2 \%$ testosterone cream can be applied to the vulva prior to sexual activity in those who complain of decreased genital sensitivity and delayed orgasm, though its effectiveness remains anecdotal. Since many patients are on antidepressants, management of SSRI-induced orgasmic delay will often be warranted. Options include waiting for tolerance to develop, reducing the dosage, switching to a different antidepressant like the dopamine-agonist bupropion, or taking drug holidays on the weekends. Augmenting SSRIs with $5-\mathrm{HT}_{2}, 5-\mathrm{HT}_{3}, \alpha_{2}$-adrenergic receptor antagonists, PDE5 inhibitors, and dopamine receptor agonists has been proposed. Such drugs include buspirone, Sildenafil, and yohimbine and may show potential once large-scale studies are undertaken. However, their safety in combination with SSRIs is currently in question. Because genital sexual arousal and orgasm depend on adequate nerve conduction involving the pudendal, pelvic, hypogastric, and sensory vagus nerves, gynecologic surgeons should be careful to spare those nerve pathways.

Patients with primarily nonmedical causes of orgasm disorder should be educated about the importance of clitoral and G-spot stimulation and the relative insensitivity of the back two-thirds of the vagina for the average woman. Women tend to learn how to achieve orgasm most easily through their own masturbation, followed by teaching these techniques to their partners. Furthermore, patients should be taught Kegel exercises as vaginal muscle strength is correlated with orgasmic potential. The stronger her vaginal muscles, the more likely the woman is to be orgasmic with intercourse. The patient should be informed that most women do not easily reach orgasm with intercourse and that only with effective stimulation and communication will she find out whether she will be coitally orgasmic. Books written for anorgasmic women can be recommended. Preorgasmic women need to be encouraged to engage in prolonged stimulation, perhaps 30-60 minutes, to break through the emotional barriers to responding. Vibrators work for most preorgasmic women, but learning to achieve orgasm by less intense methods is preferable. Women unable to benefit from these suggestions should be referred to therapy-with their partners if possible.

\section{Vaginismus}

When a patient presents with vaginismus, physicians should proceed with the pelvic examination very slowly, if at all, ideally using a pediatric speculum. 
Sometimes the patient is so phobic that an internal examination to rule out physical causes is inadvisable until the patient has benefited from psychotherapy. If the patient has other symptoms indicative of a pelvic floor disorder, such as painful defecation, then referral to a pelvic floor physical therapist is also indicated. Vaginismus is sometimes accompanied by pelvic pain disorders such as vulvodynia. The physician should be thorough in ruling out and/or treating these medical precursors to vaginismus, although sometimes a combined approach has been shown to be effective.

Physicians should educate the patient with nonmedical vaginismus symptoms by explaining the spasm of paravaginal muscles and stressing that she can learn to relax them. Physician-assigned relaxation exercises and Kegel exercises followed by the insertion of lubricated dilators of graduated circumference may be all that is needed in milder cases. Dilator insertion may be done by the physician in multiple office visits or at home by the patient and her partner. However, vaginismus is often associated with such a phobic element that a psychotherapy background is needed to treat it successfully. A combination of sex therapy and pelvic floor physical therapy has been shown to be highly effective. Assuring the patient that pain is not involved will help her take that first step.

\section{Sexual Aversion/Phobia}

This condition should be treated by a psychotherapist because of the complexity involved. If the patient is amenable, rule out both physical causes of dyspareunia via a pelvic examination as well as medical causes of low sex drive. Psychotherapy, often involving treatment for sexual abuse, and couples therapy may be warranted.

\section{Referral}

Because sexual disorders may require diagnosis and management beyond the usual training and focus of the obstetric-gynecologic clinician, it is important to develop referral sources in internal medicine, endocrinology, urogynecology, psychiatry/psychotherapy, and pelvic floor physical therapy. For a listing of pelvic floor physical therapists in your area, call The American Physical Therapy Association at 800-999-2782 and ask for the "women's health division." For nonmedical causes of sexual dysfunction, the most effective referral is to a psychotherapist who has been extensively trained to do sex therapy and couples therapy. Since Florida is the only state that currently licenses sex therapists, the most reliable credential of a good sex therapist is certification by a national organization. Currently, the American Association of Sexuality Educators, Counselors, and Therapists (AASECT) is the oldest, largest, and most nationally recognized certifying organization for sex therapists. Referrals can be reached online at www.aasect.org or by calling the national office at 804-7520026. Likewise, clinical members of the American Association for Marriage and Family Therapy (go to www.aamft.org for a list of clinical members) will have training in couples therapy, although not necessarily in sex therapy. 
Therapists from all disciplines-psychiatry, social work, psychology, marriage and family therapy, and psychiatric nursing — can be credentialed in these areas with the appropriate training.

The referral will be more effective if the clinician (1) can assure the patient that this therapist has been effective with other patients; $(2)$ reassures the patient that no sexual touching is done or demonstrated by the therapist; (3) knows that this therapist is comfortable with the subject and will not judge the patient; (4) stresses that the patient's partner (if she has a steady, committed one) often needs to be involved in the therapy for therapy to be most effective; and (5) assures the patient that the therapist will make every effort to work within the patient's value system.

\section{Case Study}

Tara, a 31-year-old working mother of two toddlers, was married for 5 years. She was referred to sex therapy for a total loss of sex drive with onset after her first delivery 3 years earlier. In addition to her loss of sex drive, she was usually anorgasmic, though she was able to achieve orgasm with coital stimulation within 5 minutes of penetration and within 1 minute of vibrator stimulation about two times a month. In addition, she reported that she resented her husband's initiations. She was taking low-dose desogesterel containing oral contraceptives for ovarian cysts, Zyrtec, and methyltestosterone for her low drive. She complained of excessive fatigue, which she attributed to raising small children. The only benefit she obtained from methyltestosterone was the eradication of her dyspareunia and restoration of vaginal lubrication. Her initial laboratory results revealed a very low total and free testosterone. (No other laboratory results were reported but there were no additional symptoms of other endocrine malfunctions.)

Tara's husband was involved in the initial evaluation but was unable to attend therapy due to work conflicts. However, the couple seemed to have a warm, cooperative marriage and she appeared to have no substantial psychopathology. Tara's history included some features that clinicians often correlate with sexual repression in adulthood. As a child she had been forced to watch her babysitter sexually stimulate her little brother and felt guilty for not protecting him. She also reported that her father was unusually negative about anything to do with sex throughout her rearing.

Despite having been informed of the probable effect of her oral contraceptives on her free testosterone, Tara chose to remain on them because of significant consequences from prior ovarian cysts. Her methyltestosterone was discontinued and she was started on a regimen of testosterone injections every 3 weeks coinciding with weekly psychotherapy. After stabilizing her dosage in the physician's office, she continued with at-home injections. Tara began to notice a positive change after the second injection, and by the fourth month her drive had substantially risen. She began to initiate sex and was receptive to her husband's initiations most of the month. She began to achieve 
orgasm in $100 \%$ of her sexual encounters, and felt comfortable with proposing a variety of sexual acts. In psychotherapy Tara worked through her traumatic feelings from the sexual abuse incident and confronted her father on the negative impact of his sexual attitudes. She learned a variety of sexual techniques and was reassured of their normalcy.

However, by the fourth month, her total testosterone ( $\mathrm{T}$ ) was rising above physiologic range though her free T remained in midrange. She lowered her dosage at her endocrinologist's request, reducing her total testosterone to normal range and her free $\mathrm{T}$ to just below normal range. After 2 months on the lower dosage, she had maintained her level of sexual desire and responsivity, felt emotionally satisfied with her sexual relations, and satisfactorily terminated psychotherapy. At no time did she complain of any unwanted side effects.

Tara's case illustrates how the combination of androgen insufficiency and sexual trauma and repression can cause sexual dysfunction at all stagesdrive, arousal, orgasm, and satisfaction. The combination of testosterone replacement and psychotherapy restored her to consistently satisfactory sexual functioning.

\section{Summary}

Sexual dysfunctions can be caused by organic, psychogenic, or mixed etiologies. Obstetric-gynecologic clinicians are often the frontline in diagnosing and treating organic causes of female sexual disorders and are crucial in referring patients whose disorders involve psychogenic and mixed etiologies. Too often, clinicians use their own experiences and value systems to guide the treatment of sexual issues, an approach that may delay effective treatment. Scientifically based knowledge is essential in effective management and collaboration with related specialists is often warranted. Women's sexual medicine suffers from a lack of adequate diagnostic techniques, laboratory tests, and treatment norms. Some treatment regimens, especially androgen replacement therapy, are still considered experimental and lack well-done studies with long-term safety data to back up clinical experience. However, research in this area is rapidly increasing and clinicians are encouraged to obtain further training by attending meetings hosted by the American Association of Sexuality Educators, Counselors, and Therapists-AASECT (www.aasect.org), The International Society for the Study of Women's Sexual Health-ISSWSH (www.isswsh.org), The Society for Sex Therapy and Research-SSTAR (www.sstarnet.org), and The Society for the Scientific Study of Sexuality-SSSS (www.sexualscience.org). Furthermore, by joining these organizations, the clinician will also have access to publications and Web-based case discussion groups. Those with a special interest in treating female sexual dysfunction are encouraged to obtain certification from The American Association of Sexuality Educators, Counselors, and Therapists. When done with up-to-date, scientifically based information, obstetricgynecologic clinicians can play an educational and therapeutic role in the lives 
of their patients with sexual problems. Those who do will find it a rewarding area of medicine.

\section{Reading for the Female Patient}

Barbach L. For Yourself. 1976 (for orgasm disorders).

Ellison CR. Women's Sexualities. 2000 (for general understanding).

Foley S, Kope S, Sugrue D. Sex Matters For Women-A Complete Guide for Taking Care of Your Sexual Self. 2002.

Goldstein A, Brandon M. Reclaiming Desire: 4 Keys to Finding Your Lost Libido. 2004.

Heiman J, LoPiccolo J. Becoming Orgasmic. 1988 (book and video).

Katz D, Tabisel RL. Private Pain. 2002 (for vaginismus).

Ladas A, Whipple B, Perry JD. The G Spot: And Other Discoveries About Human Sexuality. 2005.

Maltz W. The Sexual Healing Journey. 1991 (for sexual abuse survivors).

McCarthy B, McCarthy E. Rekindling Desire: A Step by Step Program to Help Low-Sex and No-Sex Marriages. 2003.

Zilbergeld B. The New Male Sexuality—Revised Edition. 1999 (for the male partner).

\section{Additional Suggested Web Sites}

www.aasect.org-listing of certified sex therapists

www.SexualHealth.com-for sexuality information

www.vaginismus@yahoogroups.com—for vaginismus patients only

www.lvaginismus@yahoogroups.com-for vaginismic patients, their partners, and treating professionals

www.sexualitytutor.com-links to vaginismus information, especially nonEnglish-speaking patients

See list within Maurice W. Sexual Medicine in Primary Care (referenced below)

Acknowledgments. The author would like thank Beverly Whipple, Ph.D., R.N. and Michael Plaut, Ph.D. for their editorial input. Note: Dr. Koehler was a paid consultant of Procter \& Gamble on their third stage clinical trials of the testosterone patch in women and a paid consultant on female sexual dysfunction for Ortho-McNeill.

\section{Suggested Reading}

Allolio B, Arlt W. DHEA treatment: myth or reality? Trends Endocrin Metab 2002;13(7):288-94. 
Androgen Insensitivity in Women. The Princeton Conference. Fertil Steril $2002 ; 77(4)$.

Arlt W, et al. Dehydroepiandrosterone replacement in women with adrenal insufficiency. N Engl J Med 1999;341(14):1013-20.

Barrett-Conner E. Efficacy and safety of estrogen/androgen therapy-menopausal symptoms, bone, and cardiovascular parameters. J Reprod Med 1998(suppl);43(8):746-52.

Barrett-Connor E, et al. A two-year, double-blind comparison of estrogenandrogen and conjugated estrogens in surgically menopausal womeneffects on bone mineral density, symptoms, and lipid profiles. J Reprod Med 1999;44(12):1012-20.

Basson R. The complexities of female sexual arousal disorder. World J Urol $2002 ; 20(2): 119-26$.

Basson R, et al. Report of the International Consensus Development Conference on Female Sexual Dysfunction: Definitions and Classifications. J Urol 2000;163(3):888-99.

Basson R, et al. Summary of the Recommendations on Sexual Dysfunctions in Women. J Sex Med 2004;1(1):24-34.

Berman J, Bassuk J. Physiology and pathophysiology of female sexual function and dysfunction. World J Urol 2002;20:111-18.

Castelo-Branco C, et al. Comparative effects of estrogens plus androgens and tibolone on bone, lipid pattern and sexuality in postmenopausal women. Maturitas 2000;34(2):161-8.

Crenshaw T, Goldberg J. Sexual Pharmacology: Drugs That Affect Sexual Function. New York: Norton; 1966.

Davis S, et al. Endocrine aspects of female sexual dysfunction. J Sex Med 2004; $1(1): 82-6$.

Geiger AM, Foxman B. Risk factors for vulvovaginal candidiasis: a case-control study among university students. Epidemiology 1996;7(2): $182-7$.

Guay A. Androgen values in premenopausal women without sexual dysfunction. Podium \#6. Proceedings of the International Society for the Study of Women's Sexual Health Annual Meeting. 2002.

Hatzichristou D, et al. Clinical evaluation and management strategy for sexual dysfunction in men and women. J Sex Med 2004;1(1):49-57.

Hellberg D, Zdolsek B, Nilsson S, Mardh PA. Sexual behavior of women with repeated episodes of vulvovaginal candidiasis. Eur $J$ Epidemiol $1995 ; 11(5): 575-9$.

Johannsson G, et al. Low dose dehydroepiandrosterone affects behavior in hypopituitary androgen-deficient women: a placebo-controlled trial. J Clin Endocrinol Metab 2002;87(5):2046-52. 
Leiblum SR, Nathan SG. Persistent sexual arousal syndrome: a newly discovered pattern of female sexuality. J Sex Marital Ther 2001;27(4): $365-80$.

Leiblum S, Wiegal M. Psychotherapeutic interventions for treating female sexual dysfunction. World J Urol 2002;20(2):127-36.

Lewis CE. The 60 second interview (video). Los Angeles: UCLA Center for Health Promotion and Disease Prevention; 1993.

Maurice W. Sexual Medicine in Primary Care. St. Louis: Mosby; 1999.

Michael RT, et al. Sex in America. Boston: Little, Brown \& Co.; 1994.

Munarriz R, et al. Androgen replacement therapy with dehydroepiandrosterone for androgen insufficiency and female sexual dysfunction: androgen and questionnaire results. J Sex Marital Ther 2002;28(s):165-73.

Segraves RT, Balon R. Sexual Pharmacology: Fast Facts. New York: W.W. Norton; 2003.

Simon JA. Safety of estrogen/androgen regimens. J Reprod Med 2001; 46(3):281-90.

Sipski M, Alexander C. Sexual Function in People with Disability and Chronic Illness. Gaithersburg, MD: Aspen Publications; 1997.

Whipple B. Beyond the G spot: new research on human female sexual anatomy and physiology. Scand J Sexol 2000;3(2):35-42.

Whipple B, Gerdes CA, Komisaruk BR. Sexual response to self-stimulation in women with complete spinal cord injury. J Sex Res 1996; $33(3): 231-40$.

Zaviacic M. The Human Female Prostate. Brastislava: Slovak Academic Press; 1999. 